\title{
La rhétorique de la science : Pouvoir et devoir dans un article de science exacte
}

\author{
Bruno Latour \\ Ecole des mines (Paris) \\ Paolo Fabbri \\ Université de Bologne
}

Le présent texte, reproduit ici avec l'autorisation de l'éditeur et des auteurs, est paru en 1981 dans la revue Actes de la recherche (Paris)*. Il constitue un «classique " parmi les travaux de philosophes et de sociologues qui ont porté un regard critique sur les textes scientifiques. L'analyse présentée se situe au croisement des études sémiotiques ou textuelles françaises appliquées jusquelà aux œuvres littéraires, d'une part, et de la sociologie des sciences, surtout anglo-saxonne, d'autre part. Les auteurs procèdent à une étude fouillée d'un texte représentatif de la production scientifique et montrent comment ce texte est construit et a recours à diverses stratégies de façon à argumenter et à convaincre. Dans un post-scriptum, bref mais fort intéressant, les premiers éditeurs ont réuni un résumé des objections rę̧ues par les auteurs.

On dit souvent que la science est « l'ensemble des énoncés vrais " (Wittgenstein) et que le style scientifique se caractérise par des énoncés impersonnels tels que : « la substance A agit sur la substance B ». Définir ainsi le style scientifique, c'est confondre la science telle que la présentent les manuels d'enseignement avec les écrits scientifiques qui s'échangent à l'intérieur du champ scientifique. Au lieu d'analyser le style scientifique sous ses formes vulgarisées, nous voudrions lire et faire lire un article qui se situe à la " frontière " d'une science. Notre but n'est pas de proposer une théorie du style scientifique mais d'inviter les philosophes et les sociologues à an-alyser les textes scientifiques tels qu'ils sont réellement produits. Nous avons choisi pour ce faire un article de neuroendocrinologie paru en 1962 dans les Comptes rendus de l'Académie des sciences de Paris ${ }^{* *}$. Si cet article a été choisi comme matériel privilégié, 
c'est qu'il constitue la principale production d'un laboratoire dont les articles sont la production essentielle (la production annuelle d'un groupe de dix docteurs est de quarante articles en moyenne). On compte un article de vulgarisation pour quarante de «frontière ». En dehors des articles, le laboratoire produit des communications informelles de chercheurs à d'autres chercheurs, des chercheurs et des techniciens formés sur place, des substances communiquées pour étude à d'autres chercheurs.

L'analyse que nous présentons est au croisement d'une étude de sociologie des sciences et d'une étude de sémiologie. La sociologie des sciences, surtout anglo-saxonne, utilise depuis longtemps les articles comme unité de calcul pour définir la productivité des chercheurs ou pour dessiner les limites et mesurer la croissance des disciplines'.

Les publications de l'Institute for Scientific Information, à Philadelphie, et en particulier le Science Citation Index qui donne chaque année pour chaque article le nombre de citations dont il a été l'objet (il en existe aussi un pour les sciences humaines) fournissent le matériau de nombreuses études de sociologie de la science (la revue Current Contents donne une revue hebdomadaire de cette littérature).

Dans toutes ces recherches seules les citations sont considérées, jamais le contenu et encore moins le style ${ }^{2}$. De son côté l'analyse sémiotique étudie les formes de récit mais n'applique pas les méthodes qu'elle a mises au point ${ }^{3}$ à des textes de science de la

Ce tableau présente les quatre façons les plus courantes d'aborder la littérature scientifique. Pour des raisons pratiques qui seront expliquées ailleurs, la connaissance scientifique peut être considérée comme un réseau d'articles qui agissent les uns sur les autres par l'intermédiaire des savants (colonne $C$ ). Mais on peut aussi (colonne $B$ ) considérer que les savants agissent les uns sur les autres par l'intermédiaire des articles, obtenant ainsi la reconnaissance. Tout se passe donc comme si les articles engendraient d'autres articles et comme si les chercheurs n'échangeaient entre eux que du prestige. Telles sont les deux voies que suit alternativement la sociologie des sciences. Ces deux voies differrent de l'analyse commune qui voit dans la Science soit des Savants - quand il n'y a que des auteurs -, soit des Connaissances - quand il n'y a que des réseaux d'articles -. Les colonnes $A$ et $D$ du tableau résument ces deux façons de faire disparaître l'activité scientifique soit dans des individus, soit dans des énoncés. Dans cet article nous suivrons une voie, différente bien sûr des deux analyses communes, mais différente aussi des deux voies suivies jusqu'ici en sociologie des sciences puisque nous considérons à la fois les stratégies des auteurs et les interactions des textes (ligne brisée du schéma). 
Images hagiographiques et images sociographiques de la science

"SAVANT" CONNAISSANCE

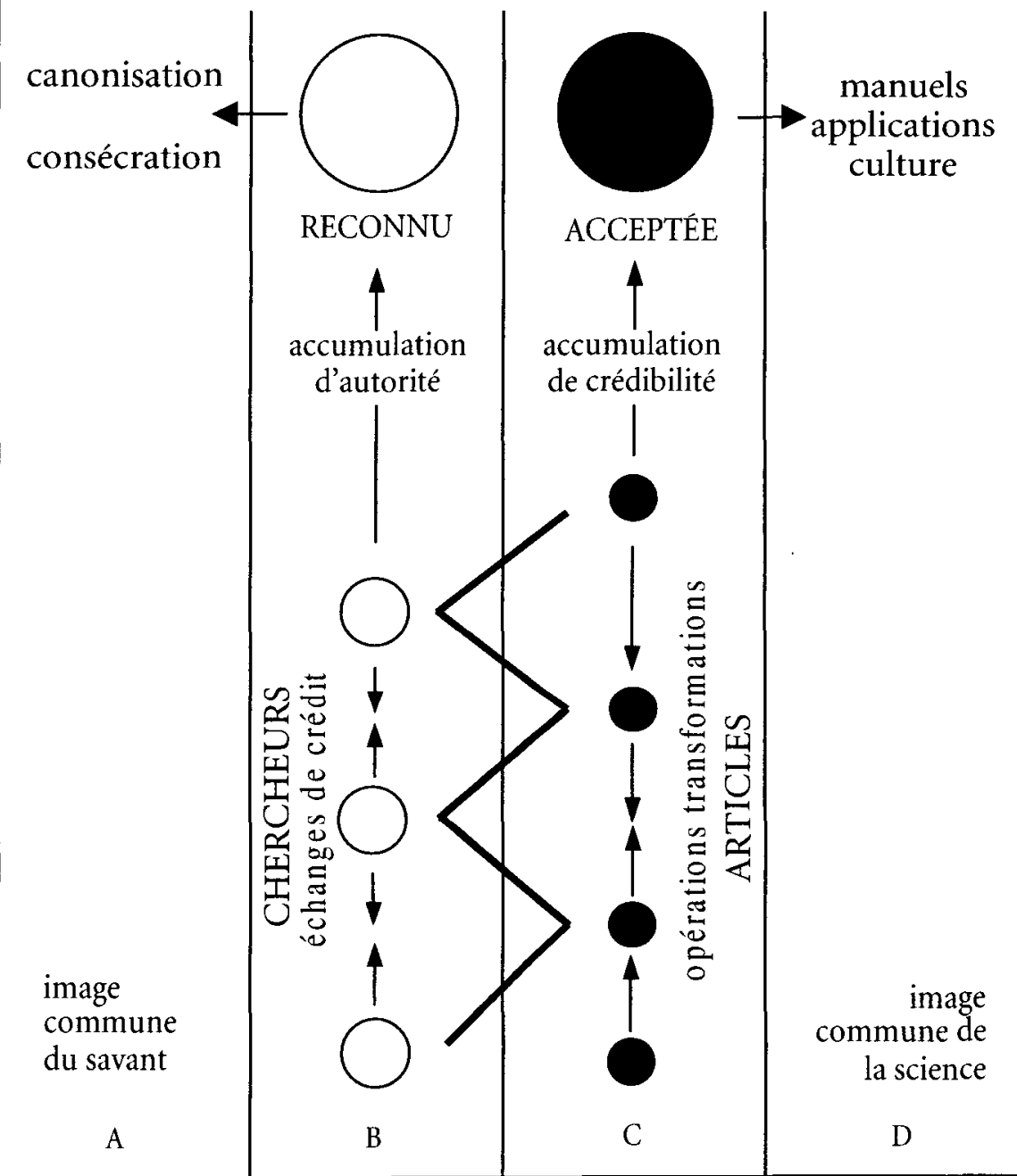


nature bien que plusieurs tentatives aient été faites en ce qui concerne le discours des sciences humaines ${ }^{4}$. Il était donc intéressant de mener la sociologie des sciences au cour des articles, grâce à l'analyse littéraire moderne et de vérifier par là si la littérature des sciences exactes obéit à des règles générales, valables pour toute littérature.

\section{Les marqueurs sociologiques}

L'article que nous présentons porte comme tous les autres la marque des différents éléments dont le sociologue cherche à peser l'influence respective. Six marques dans ce texte renvoient explicitement aux conditions de sa production. Le mot " Endocrinologie " permet d'accrocher l'article par un mot-clef dans les différents systèmes de classification. Pourtant cet article appartient à la neuroendocrinologie, discipline vieille de trente ans, au croisement de deux autres, et qui n'est pas encore assez établie pour avoir son propre index dans les Comptes rendus, bien qu'elle ait ses journaux, ses chaires et ses congrès. Par ce mot se marque dans le texte l'histoire par laquelle les disciplines s'ouvrent, s'institutionnalisent et dessinent leurs limites ${ }^{5}$. L'ordre choisi pour présenter les quatre auteurs reflète l'état des rapports de force au sein du groupe - rapport qui intéresse beaucoup les sociologues américains et renvoie à toute la microsociologie d'une équipe de recherche $e^{6}$.

Un astérisque renvoie au « 23 juillet 1962 », date de la séance de l'Académie des sciences lors de laquelle ce papier fut présenté7. Cette date, différente de la date de publication, permet de résoudre certaines querelles de priorité. Comme il n'est pas d'idée, à la frontière d'une science, qui ne soit datée, le délai entre la date de réception et la date de publication est un facteur important dans le choix d'un journal. Les Comptes rendus sont d'ailleurs connus pour publier en quelques semaines. La « Note», datée, titrée, appropriée et indexée est de plus " présentée par M. Robert Courrier ». C'est en effet par cet académicien que cette Note dut passer pour être lue devant les pairs. Il est un de ceux qui autorisent ou non les auteurs à paraître et que les anglosaxons appellent gate-keepers. Leur pouvoir est souvent considérable. À la page 5 de l'article, deux autres marques réfèrent l'une aux fonds de recherche, l'autre à l'institution qui ont rendu cette recherche possible. L'argent n'est pas signalé par son montant mais par le numéro de la subvention (grant). Ce numéro renvoie au système compliqué par lequel la proposition du groupe a été évaluée par un groupe de pairs et acceptée ; dans ce cas précis à la fois par les fonctionnaires du ministère américain de la santé et, du côté français, par le Centre national de la recherche scientifique. Le Collège de France est l'institution qui a accepté de procurer au groupe l'espace physique et administratif. Mais l'espace est un espace socialisé qui est de plus un bien 
rare. Autour de lui se noue toujours un réseau de calculs et parfois d'intrigues : pourquoi tant d'espace pour ce chercheur? pourquoi l'accès à tel instrument? pourquoi telle facilité administrative?

Ainsi, avant même d'avoir lu l'article, le sociologue des sciences se trouve devant un objet très riche qui renvoie explicitement à un double jeu de stratégies : celui de l'auteur (choix de la discipline, choix du titre, choix du moment, choix du journal) et celui des institutions (choix de l'autorisateur, choix des bailleurs de fonds, choix du laboratoire). L'article renvoie aussi implicitement à tout un jeu de conflits qui n'apparaissent pas comme tels dans le texte. C'est à l'intersection de toutes ces pratiques que se situe la production de ce morceau de connaissance. Qu'une seule de ces conditions de production varie et l'article que nous avons sous les yeux serait différent.

\section{Rhétorique et communication}

Comme nous allons utiliser dans la lecture du texte quelques termes qui ne sont pas encore d'usage tout à fait courant, arrêtons-nous quelque temps pour les définir. Il faut introduire une distinction entre la dimension " pragmatique " du texte - qui l'écrit, pour qui et pourquoi - et sa dimension proprement textuelle. Mais dans le texte lui-même il faut distinguer le système " d'énonciation " par lequel l'auteur choisit ou non d'introduire la dimension pragmatique, et le système des " énoncés " qui ne fait aucune référence à l'auteur. Dans le tableau des énoncés nous avons récapitulé toutes les marques par lesquelles l'auteur s'annonce dans le texte. Contrairement à l'opinion répandue on voit que ce texte, bien que scientifique, n'a rien d'impersonnel. Dans les énoncés il faut distinguer les propositions et les " modalisations » entendues comme tout ce qui modifie une proposition. Enfin par « anaphore " nous désignons tout renvoi du texte à lui-même ou à d'autres textes.

Le trait le plus marquant de ce texte apparait dès qu'on en survole le contenu : il est hérissé de références. La page 5 de la Note groupe dix références qui dessinent le contexte de l'article. Les sept premières renvoient à des écrits déjà publiés qui vont être travaillés par la Note, les trois dernières à des méthodes qui sont abrégées par un chiffre : « préparé comme dans ${ }^{8}$ », ou « adaptée de la méthode de Mc Kenzie ${ }^{9}$ ». Toutes ces références signalent des œuvres à l'extérieur du texte mais d'autres opèrent à l'intérieur. Ainsi tout le paragraphe 5 se réfere à la table de la page 3 . Cette table ellemême renvoie à la figure de la page 4 . Cette table et cette figure sont obtenues dans le laboratoire à partir des instruments. Elles sont, pour ainsi dire, l'écriture symbolique des instruments et composent l'infratexte sur lequel la démonstration va s'établir. Par une troisième espèce d'anaphore les paragraphes 1,2 et 6 - que nous désignerons dorénavant comme texte $\mathrm{A}$ - renvoient aux paragraphes 3,4 et 5 - texte $\mathrm{B}$. Ainsi les 


\section{Ênonciation et énoncés}

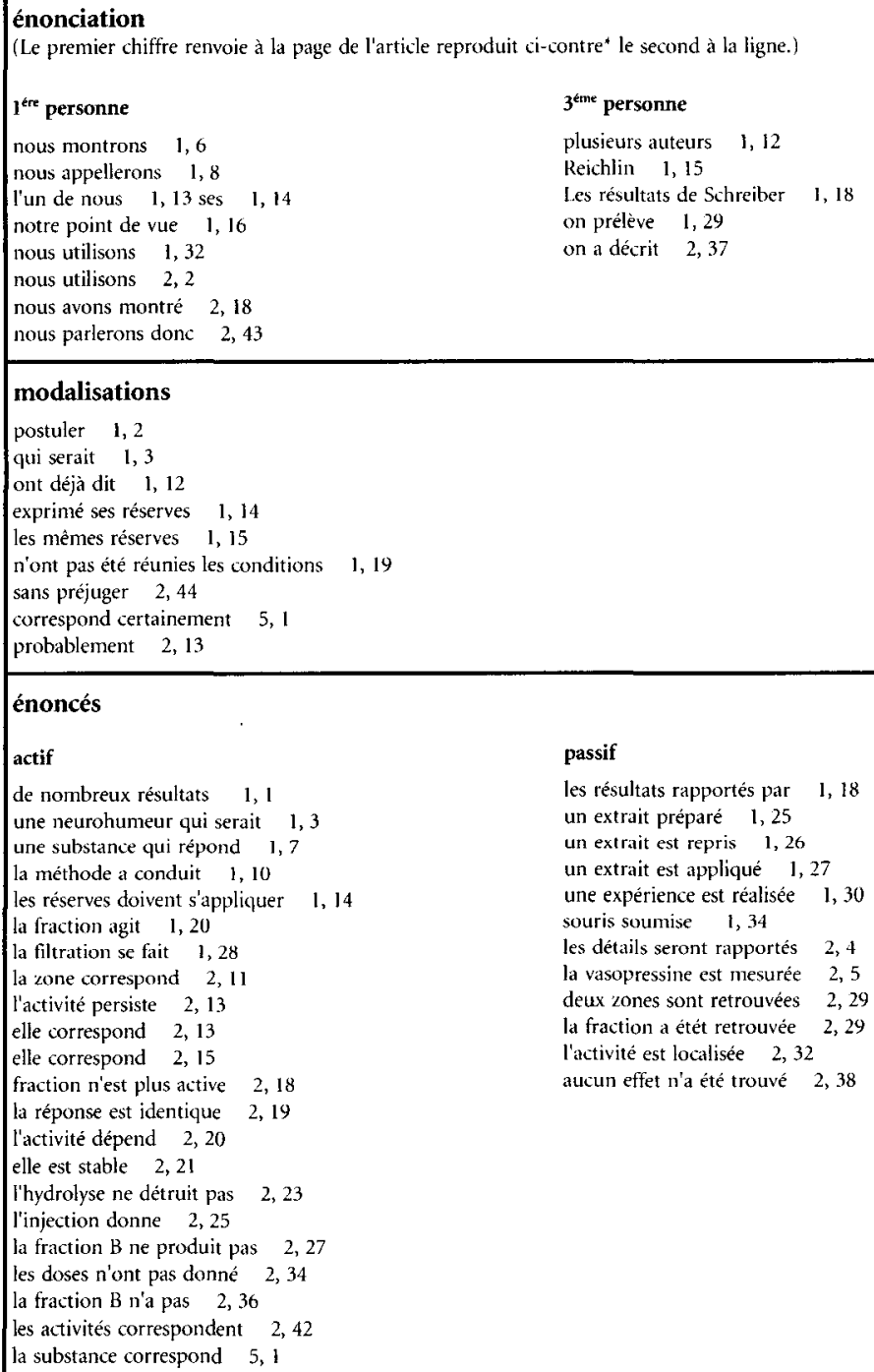


expressions «par cette Note nous montrons " (p. 1, ligne 5) et «les activités décrites plus haut » (p. 2, 1. 40), montrent que le texte A, lui-même agissant sur le contexte, est fondé par référence au texte $B$ qui est lui-même permis par l'infratexte. La littérature des instruments va permettre de transformer la littérature publiée. Enfin, quatrième espèce d'anaphore, le texte est dans son entier repris par le titre et le plus souvent, mais pas dans ce cas, par un sommaire (abstract). Le titre ou le sommaire n'indique pas (ou n'évoque pas) simplement le texte, il en concentre toute l'information, chose rare en sciences humaines et impossible en littérature. La multiplication de ces références modifie le problème classique du référent (par référent nous entendons simplement de quoi parle un discours quelconque). Dans d'autres pratiques littéraires ou bien il y a un référent qui n'est pas textuel ou bien il n'y a pas de référent du tout cas de la fiction voulue comme telle. Or, dans l'article que nous présentons, il y a bien un référent, mais celui-ci est composé d'un empilement de textes : le contexte sur lequel l'article agit, l'infratexte sur lequel il s'appuie, la partie B sur laquelle se fonde la partie A. Tout se passe comme si la solidité du papier, d'autres diraient son objectivité, venait des correspondances établies en repliant l'une sur l'autre ces différentes couches de textes. Ce n'est pas la nature que l'on trouve sous le texte scientifique, c'est la littérature des instruments.

\section{Le problème de l'information transmise}

Ce n'est pas la nature (référent ultime) que l'on trouve en aval ou en amont du texte, mais d'autres textes encore qui le citent ou qu'ils citent. D'autres formes d'écriture ont pour effet soit de concentrer l'attention sur un objet unique et irremplaçable (cas du roman ou du poème) soit de transmettre de l'information (cas du manuel, de l'annuaire, etc.). Or cet article est, par définition, remplaçable puisqu'on doit pouvoir construire sur lui. S'il est vrai, il est repris en une demiphrase dans un autre article ; s'il est faux, il peut être, soit repris dans un autre pour être contesté, soit complètement ignoré. ${ }^{8}$. Pas plus qu'il ne ressemble à un roman cet article ne ressemble à un annuaire. En fait la quantité d'information transmise par ces pages est faible si on laisse de côté le cas du paragraphe 3. Il ne faudrait pas prendre pour de l'information l'ensemble des mots techniques — " diencéphale », " TRF », " acétate de pyridinum " - car ils désignent justement ce qui, pour le petit groupe de pairs auquel l'article est destiné, ne fait pas question. Les termes « barbares » peuvent rendre le texte obscur au lecteur non informé mais n'apprennent rien de nouveau aux lecteurs scientifiques. Quelle est donc la nouvelle information transmise qui justifie que le texte ait cinq pages? Ce texte ne transmet pas d'information; il agit. Pendant cinq pages il cherche à convaincre. De quoi ? Qu'un mouvement important, qu'une 
opération importante a été faite dans la littérature. Qui cherche-t-il à convaincre ? Le groupe des pairs, par définition le plus difficile à convaincre et composé de gens mis en cause par le " coup ». Comment va-t-il chercher à convaincre ?

Par d'autres textes produits dans le laboratoire et « produits » ici comme preuve. C'est l'analyse de ce " coup " - au sens politique, sportif ou ludique du terme - qui va nous permettre d'entrer dans le contenu de l'article. L'opération effectuée par l'article est résumée dans le titre et explicitée dans le paragraphe 1. Dans la première phrase le sujet parlant (i.e. le ou les auteurs) est remplacé par un impersonnel (" de nombreux résultats ") qui " ont conduit " à la modalisation d'une proposition. La modalisation est marquée deux fois, d'abord par le verbe " postuler ", puis par l'emploi du conditionnel (" qui serait "). La proposition pourrait se traduire en langage courant : il existe dans l'hypothalamus une sub-stance qui contrôle la sécrétion par l'hypophyse de l'hormone TSH, laquelle à son tour, règle la sécrétion des hormones de la thyroïde? L'action du texte se traduit par une double transformation dans la deuxième phrase. À la place de l'impersonnel (" de nombreux résultats") apparaît le sujet parlant: " nous montrons"; à la place de la modalisation (" postuler l'existence d'une substance qui serait") apparait l'affirmation : "l'existence d'une substance qui correspond aux caractéristiques attendues ». Par la mise en scène de cette double transformation une possibilité discutée devient une existence indiscutable. Pour comprendre ce mouvement il faut en saisir l'enjeu. Depuis vingt ans un paradigme s'était constitué à partir des données de la physiologie selon lequel le système endocrinien, loin de se régler lui-même (feed-back) pourrait être contrôlé par le système nerveux, via l'hypothalamus. Ce paradigme n'est plus contesté dans les années soixante. Mais comme l'ontologie implicite de l'endocrinologie est résolument substantialiste, un sous paradigme devait être établi, posant l'existence de substances qui seraient, comme chez Aristote, homogènes, spécifiques et discrètes. Or, jusqu'à cet article, la neuroendocrinologie présente un chaos de résultats, de prétentions, de preuves et de contrepreuves, mais aucune substance nettement identifiée. Cet article, au contraire, ou plus exactement, le groupe d'articles qui l'entoure - construit un objet au contour net ; c'est du moins ce qu'il dit et ce que les autres textes qui le citent reconnaissent. Tout texte cherche à convaincre, mais le texte scientifique obtient cet effet en développant des couches de textes qui se correspondent et qui servent l'une à l'autre de référent. Ici par exemple, le bioétalonnage, les étapes de la purification, les règles de la méthode, l'analyse statistique, tout concorde. Cette concordance rompt avec les discordances qui la précèdent. Où il y avait des prétentions il y a maintenant un objet. Pour réaliser l'enjeu de ce mouvement, il faut savoir qu'il a fallu plus de huit ans, une centaine d'articles, plusieurs millions de dollars et une demi-douzaine de personnes pour passer de cet article de 1962, qui montre l'existence de cette substance, à un 
article de 1969 dans lequel sa formule chimique est démontrée (Comptes rendus Académie des sciences, t. 269, 12.9.1969). Le texte de 1962 vérifie le paradigme, le concentre en trois mots ( "de nombreux résultats ") et une référence ( "voir la récente revue de Bogdanove (1) », p. 1, 1.5). Par cette action le champ d'argumentation qui le précède est rendu obsolète. Un nouveau champ commence que l'on pourrait intituler " isolation et caractérisation du TRF ». Lorsqu'en 1969 la formule chimique de cette substance est établie, toute la littérature produite pendant huit ans devient à son tour obsolète. Toutes les études partent dorénavant de ce papier final et ouvrent à partir de lui d'autres champs. Le champ de discussion peut être défini par une stricte intertextualité, une centaine de papiers qui font explicitement référence les uns aux autres et disparaissent tous dans le dernier.

\section{Le détour par la polémique}

La position que l'auteur veut emporter est déjà prise. " Plusieurs auteurs ont déjà dit avoir mis en évidence et purifié la substance TRF " (p. 1, 1. 12). Il va donc falloir les déloger de cette position qui commande l'ouverture du champ d'étude. La contestation se fait par un effet de style (" ont dit avoir montré ") qui s'oppose bien sûr au « nous montrons" de la ligne 6. La même opération aurait pu se faire plus brutalement ou plus délicatement. Dans un article américain on aurait une remarque polie constatant que les résultats des deux laboratoires ne correspondent pas. À ce niveau les habitudes professionnelles et nationales, l'éducation personnelle et l'art de bien écrire peuvent intervenir pour moduler des opérations d'écriture qui échappent largement à leur auteur. L'étude des opérations que nous présentons ici n'est pas réductible à celle d'un style individuel. Elle renvoie dos à dos ceux qui prétendent que, dans un article scientifique, "il n'y a pas de style ", ou ceux, au contraire, qui n'y voient que le reflet d'un style particulier. Choisir l'une ou l'autre de ces positions, c'est ne pas faire la différence entre les stratégies de l'écriture scientifique imposées par l'état du savoir, les rhétoriques imposées par la communauté scientifique et les petites tactiques de style décidées par l'individu.

Suivent dix lignes de combat - agonistique ou polémique comme on voudra dans lequel des Noms-Articles ( Libert (6) ", "Reichlin (7) ") et non des personnes sont mis en cause. L'agonistique en science obéit à des règles particulières et consiste, dans cette partie, à réintroduire des modalisations dans des énoncés dont la prétention était de s'en passer. Lénoncé scientifique existe en effet sous deux formes : la forme d'énoncé simple et la forme modalisée. La réussite, si l'on peut dire, pour une affirmation de science exacte est de circuler nue, sans aucune modalité sous la forme " $\mathrm{A}$ est $B$ ». Son échec est de rester engluée dans ses modalisations (certains prétendent 
que « $A$ est $B$ " $)^{10}$. La plus sévère attaque qu’on puisse imaginer est par conséquent de tourner une assertion quelconque et de la replonger dans les conditions de sa production. Ainsi : "L'un de nous a exprimé ses réserves sur les conclusions de Shibuzawa et coll. " (p. 1,1.14), « n’ont pas été réunies toutes les conditions nécessaires pour affirmer que la fraction active de Schreiber et coll. n'agit que... » (p. 1, 1. 19). D'autres NomsArticles sont, de plus, appelés pour soutenir cette attaque : « Reichlin (7) vient d'ailleurs de confirmer notre point de vue" (p. 1, 1. 15). À la place des énoncés simples : « il existe un TRF ", "A est $B$ ", on ajoute différents types de modalisateurs, soit dans le cas d'un auteur : $X$ a dit que " $A$ est $B$ "; dans le cas d'une argumentation : les conclusions de $\mathrm{X}$ menant à l'énoncé " $\mathrm{A}$ est $\mathrm{B}$ "; dans le cas de manipulations : les expériences in vitro qui permettent à $\mathrm{X}$ de conclure que " $\mathrm{A}$ est $\mathrm{B}$ ». On prétend toujours que l'idéologie scientifique est une représentation de type théâtral qui cache les coulisses et offre au public un déroulement théorique sans personnage ni histoire. Or, dès qu'on approche de l'activité scientifique réelle, on s'aperçoit que cette idéologie n'est pas celle des savants, mais plutôt celle que les philosophes veulent leur imposer. L'opération scientifique par excellence n'est pas de cacher les conditions de production mais de les mettre à la place de la représentation que les auteurs cherchent à monter. Le résultat peut être désastreux pour l'auteur pressé qui cherche à se débarrasser le plus vite possible de toutes ces conditions. On peut même définir la frontière d'une science comme ce lieu où des énoncés sont constamment replongés par les adversaires dans les conditions expérimentales qui les ont produits. Toute science « froide » au contraire se présente comme un enchaînement d'affirmations ; aussi longtemps du moins qu'un changement de front ne vient pas mobiliser de nouveau les énoncés et révéler leur origine réelle ${ }^{11}$.

\section{Le droit de dire et le devoir-faire}

Dans l'agonistique est contesté le droit de dire (que la fraction active est attribuable à la substance TRF). L'enjeu du débat est donc l'autorité. Ils disent qu'ils ont isolé le TRF mais si l'on regarde de près leurs titres on s'aperçoit qu'ils n'en ont pas le droit. La Note, tout au contraire, a pour but d'emporter la conviction du lecteur : je pouvais ne pas croire à l'existence de la substance TRF ; maintenant je ne peux pas ne pas y croire ; je pouvais croire que Shibuzawa l'avait isolée, maintenant je ne peux plus le croire. Le transfert d'autorité est indissolublement professionnel et intellectuel. Celui qui est capable dans l'article d'accumuler assez d'autorité pour convaincre définitivement qu'il a bien montré l'existence de la substance TRF, s'assure la domination du nouveau champ d'étude. En effet, l'opération de convaincre va déclencher dans l' « âme » des pairs la reconnaissance. Autrement dit, l'auteur va recevoir du 
crédit. Ce crédit - marqué quantitativement par les citations ${ }^{12}$ - peut se convertir à l'intérieur de la profession en position et en fonds de recherche lesquels, réinvestis dans le laboratoire, vont permettre d'augmenter la « mise " et de mener la substance plus loin. Bien qu'il soit beaucoup trop tôt pour faire l'analyse complète du crédit dans les sciences exactes, on voit que le droit de dire (autorité intellectuelle) entraîne un pouvoir de convaincre qui, par l'intermédiaire de la reconnaissance qu'il suscite, fonde à son tour un nouveau droit (professionnel) de dire ${ }^{13}$. Ces questions de droit sont manifestées plusieurs fois dans notre texte par des modalités qui devraient n'avoir aucune place si la science s'écrivait comme les philosophes le disent. Il en est ainsi de « les réserves doivent s'appliquer» (p.1, 1. 15) et « toutes les condi-tions nécessaires n'ont pas été réunies " (p.1,1.19) qui s'opposent aux phrases de la ligne 7 « qui correspondent aux caractéristiques attendues » et p.2, 1. 43 « qui cor-respond aux caractéristiques hypothétiques attendues». Tout se passe comme si le texte tournait autour d'un point de droit et comme si l'accord ou le désaccord avec ce point conférait ou était l'autorité de dire.

Or, par un intéressant détour, le premier auteur de l'article a, dans un texte précédent, défini les « treize critères " qu'il faudrait avoir remplis pour pouvoir conclure que l'on a bien observé une hormone hypophysiotropique et non pas un artefact. Les treize critères définissent les règles du jeu sans lesquelles le combat qui précède aussi bien que le montage expérimental qui suit seraient incompréhensibles. Il suffit d'un coup d'œil sur le texte du Journal de physiologie ${ }^{* * *}$ pour saisir l'omniprésence des modalités déontiques (exprimant le devoir faire), comme "il faut ", " il faudrait ", "on doit s'attendre". Il ne s'agit pourtant pas d'un texte juridique qui tirerait son droit d'une loi transcendante, d'une loi naturelle ou d'un précédent. Chaque requisit est directement tiré d'une longue série d'échecs expérimentaux (dix années sur le CRF sans produire une substance au contour net), tels qu'ils sont réfléchis par quelqu'un qui, selon le mot de Nietzsche, ne veut ni tromper ni être trompé. Si l'on cherchait un équivalent politique à ce manifeste on le trouverait dans la notion, chère à Stephen Toulmin de " coup d'état » ou, même, de règlement de compte avec soimême ou avec les autres. En treize points ce texte définit les conditions de l'autorité dans un nouveau domaine. Si je veux savoir, alors je dois faire les opérations énumérées de 1 à 13 pour pouvoir dire (que $A$ est $B$ ). Le droit est la façon dont le vouloir savoir se traduit en ascèse expérimentale. Les concurrents dans le champ d'étude, au fond, ne veulent pas savoir. Du paradigme ils concluent sans grand risque qu'il doit y avoir un TRF et ils le voient en effet. Ils vivent du paradigme, ils n'ouvrent pas un nouveau champ. Mais si le vouloir est tendu comme un arc et si l'on veut mener de TRF, substance que l'on montre (article de 1962) à TRF, substance que l'on nomme chimiquement (article de 1969), alors il faut être absolument sûr qu'au point de départ 
on a bien du TRF et pas autre chose. La polémique contre les autres n'est que la conséquence d'une polémique contre soi-même, et la mesure de cette polémique est donnée par la force du vouloir. C'est parce que l'on ne s'autorisera pas à dire qu'on a vu du TRF avant d'avoir obéi aux treize critères, que l'autorité des autres va se trouver défaite. Le résultat en vaut la peine, c'est la génération d'un nouveau champ d'étude dont on sera l'autorité indiscutable.

\section{Le pouvoir de prouver}

Nous avons étudié jusqu'ici les marques sociologiques, puis les références, enfin la stratégie des mouvements que l'article effectue dans la littérature. Nous devons maintenant étudier ce qui rend possible cette stratégie. Les paragraphes 3,4 et 5 (partie B) paraissent si «techniques » qu'ils semblent irréductibles à toute analyse sociologique. Laissons de côté pour l'instant le paragraphe 4 qui n'est que l'annonce d'articles parus ou à paraître. Si l'on considère le paragraphe 5 et qu'on le superpose à la table des treize critères, on s'aperçoit que chaque phrase vient de l'un des critères et $v a$ vers la partie A du texte (paragraphes 1, 2 et 6) pour la rendre possible. Cette partie n'est donc pas plus " technique " que la première. Loin de nous donner ses protocoles et ses registres, l'auteur argumente, mais il argumente avec d'autres textes. Il apparaît donc impossible de distinguer les données qui seraient " dures " et les hypothèses qui seraient " molles ", car le montage expérimental tout entier est depuis l'origine un stratagème pour marquer un point dans le combat qui mène au convaincre. Bien malin qui maintiendra dans un tel article la différence philosophique entre l'hypothèse et la vérification. Si l'on veut trouver une différence il faudrait la chercher du côté des images militaires entre le front et l'intendance, ou du côté du jeu entre les jetons accumulés et le coup qui les risque. En conclure que les instruments sont inutiles ou que les expériences sont secondaires, c'est n'avoir jamais combattu et n'avoir jamais joué.

Le paragraphe 5 relate des épreuves qu'on a fait subir à des extraits d'hypothalamus et qui produisent des effets. Ces effets sont presque toujours des jeux d'écriture (diagramme, spectre, courbes, photos) sur des surfaces d'inscription. Les paragraphes 3 et 4 rappellent dans quel type d'instrument - le bioétalonnage - et sur quel type d' "écran " les effets sont recueillis. D'autres articles rapportent précisément comment le bioétalonnage s'est constitué. Il a fallu d'énormes efforts pour mettre au point l'écran sur lequel une image comme celle de la page 4 de l'article a pu se dessiner. C'est grâce à une combinaison d'habileté manuelle, de flair 
expérimental et d'intelligence que, pour la première fois, fut captée sur l'instrument une forme stable : le pic de retardement sur lequel se surimpose le résultat du test d'activité TRF.

C'est ce qui explique l'effet du paragraphe 3 sur le petit groupe de lecteurs. Le nombre d'hypothalami est nouveau et tranche sur les petites quantités utilisées jusqu'ici. L'utilisation de la pyridine ainsi que d'une colonne "Sephadex G 25 " introduit une méthode qui commence à peine. De même l'usage du rat au lieu de la souris dans le paragraphe 4 introduit un élément nouveau. La partie la plus importante pour évaluer le crédit des affirmations qui précèdent se trouve là. En effet tout récit scientifique est précédé d'un récit « instrumental " - au sens du mot dans l'analyse littéraire -, comme lorsqu'on décrit l'épée qui va servir à l'épreuve glorifiante du héros. Par ce récit les conditions d'accumulation du crédit (faits, résultats) sont définies. Dans le cas présent l'argument devient fort par ce seul paragraphe technique. On sait à le lire que ces nouvelles méthodes vont enfin supporter les affirmations. Toutefois cette histoire de l'instrument ne nous intéresse pas ici, puisque dans le paragraphe 5 elle est considérée comme achevée.

La page 3 de l'article donne le détail des épreuves subies, à l'intérieur de l'instrument, par les fractions purifiées. À la fin de chaque épreuve on trouve un chiffre. Ces tables ne donnent évidemment pas les résultats bruts et ne suivent pas les méandres de l'expérience réelle. Ce sont des tables de chiffres « nettoyés » et prêts à rentrer, à tout instant, dans l'argumentation. Regardons une fois de plus cet empilement si caractéristique du texte scientifique. La page 3 résume un long protocole en quelques chiffres mobilisés. Le paragraphe 5 mobilise les chiffres de la page 3 , mais aboutit à une modalisation et non à un chiffre. Le paragraphe 1 à son tour utilise les modalisations du paragraphe 5 , mais son résultat, loin d'être une modalisation, est un coup dans la littérature.

Cette accumulation de textes peut être résumée en un graphique. Les récits instrumentaux couvrent la Note, le texte des treize critères définit l'autorité. Au milieu s'empilent les couches textuelles de notre article. En amont on trouve les autres papiers qui sont transformés par l'opération du texte et en aval ceux qui le transforment. Ce caractère d'empilement explique pourquoi un laboratoire est une unité de production fort différente d'une usine. L'usine pharmaceutique produit des matières qui produisent de l'argent ; le laboratoire produit des matières, qui produisent des chiffres, qui donnent des modalisations, qui emportent la conviction. Dans le premier cas on a des substances vendues, dans l'autre des énoncés crédités. En fait ${ }^{14} l e$ jeu entre ces deux équivalents universels - l'argent comme capital et l'information est plus complexe. 


\section{L'autorité des faits}

Nous avons affirmé que le paragraphe 5 établissait des modalisations i.e., argumentait - La phrase " deux zones d'activité sont constamment retrouvées " n'est pas une simple constatation. C'est une forte modalisation qui permet de dire qu'il s'agit d'une substance et non d'un artefact. Cette constance en effet, répétée p.2, I. 29 , est justement ce qui faisait défaut aux autres démonstrations. Que des effets constants soient obtenus « à partir de l'hypothalamus » alors qu'aucun effet n'est obtenu sur «l'extrait acétique du cortex cérébral » n'est pas rapporté pour le plaisir de soumettre une curieuse information, mais pour prouver que l'expérience obéit à la loi n. 1. Par cette phrase une nouvelle objection est dénouée et le droit de dire est renforcé. L'épreuve de la table 2 (page 3 de l'article) est relatée à la ligne 25 du paragraphe. L'expérience sur laquelle elle se fonde avait été imaginée, depuis le début, pour obéir à la loi n. 3, qui demandait que la stimulation se fasse en suivant une fonction linéaire du log. de la dose injectée. Une fois de plus l'accord entre les conditions posées par la loi et celles obtenues dans l'épreuve permet de créditer d'un point l'affirmation selon laquelle le TRF est présent. Nous avons jusqu'ici employé le mot d' «épreuves » pour désigner ce qui devrait apparemment recevoir le nom d' "expérience ». L'expérience, celle qui s'est passée pratiquement dans le laboratoire avec des animaux, des bocaux et des instruments, n'est pas relatée ici, ce serait trop long et surtout inutile. À la place de l'expérience, le paragraphe 5 met en jeu des " épreuves " au sens littéraire du terme. Ces épreuves sont compliquées dans le cas présent par l'absence de leur véritable enjeu lequel se trouve dans la table des treize critères. Prenons par exemple la ligne 10. On se trouve en face de deux fractions actives. Comment distinguer la "bonne " de la "mauvaise" fraction? En leur faisant passer une épreuve, définie par la loi n.6: pour pouvoir dire qu'il s'agit d'une hormone hypophysiotrope, l'activité doit cesser après hypophysectomie. Pas un mot n'est dit de l'expérience réelle, telle qu'elle s'est déroulée dans l'espace et le temps, mais l'épreuve textuelle est claire et peut être résumée ainsi :

\begin{tabular}{|l|l|r|}
\hline fraction A & hypophysectomie & activité \\
fraction B & pas d'activité \\
\hline
\end{tabular}

La première et la troisième colonnes définissent les acteurs et la colonne centrale l'action ou dans ce cas l'instrument. Mais il est clair que l'épreuve, si on l'arrête à ce point, est dépourvue de toute signification. Son sens n'est visible que si l'on ajoute à droite une seconde épreuve, celle-ci de conviction : 


\begin{tabular}{|c|c|c|c|c|}
\hline $\begin{array}{l}\text { fraction } \\
\qquad A \\
\text { fraction } \\
\text { B }\end{array}$ & hypophysectomie & $\begin{array}{l}\text { activité } \\
\text { pas } \\
\text { d'activité }\end{array}$ & requisit n. 6 & $\begin{array}{c}\text { ne peut en aucun cas } \\
\text { être TRF } \\
\text { peut sans contradiction } \\
\text { être TRF }\end{array}$ \\
\hline
\end{tabular}

Il serait fastidieux de reprendre ainsi tout le paragraphe mais chaque phrase a la même organisation. La fraction B est jugée différente de la TSH pour obéir à la loi n. 10, de la vasopressine, de l'oxytocine et enfin du TSH retard. À chaque fois c'est, dans la tête du lecteur informé, un argument qui s'effondre, une réticence qui se renforce, une objection qui se dénoue. Ce paragraphe n'est pas « technique »; c'est une discussion dont la forme particulière est faite d'une triple référence aux tables de la page 3, à la table des 13 règles et à la partie $\mathrm{A}$ du texte. Il s'agit, en d'autres termes, d'une forme particulière de rhétorique.

La première phrase du paragraphe 6 qu'il nous reste à étudier, vérifie amplement le point de vue que nous avons adopté. Tout se passe comme si elle totalisait les effets de conviction produits par chaque phrase du paragraphe 5 . «Les activités décrites plus haut $(. .$.$) correspondent aux caractéristiques hypothétiques attendues de la$ neurohumeur TRF » (p. 2, 1. 43). La boucle est bouclée, le « coup » est fait : le nom TRF désigne maintenant dans l'esprit des lecteurs-concurrents un objet net. Huit ans après il changera de nouveau de contenu et se mettra à désigner Pyroglu-His-ProNH2. Aux deux modifications de signifiés le nom de l'auteur principal, à la fois individu et groupe, se trouvera si bien attaché, qu'une sorte d'éternité lui sera dévolue. Pourtant l'article ne s'arrête ni sur les totaux qui permettent de créditer l'assertion, ni sur un cri de triomphe. Il finit au contraire sur une opération de prudence. À la l. 40, p. 2, on peut lire que les activités sont " attribuables à une ou plusieurs substances ". Aussitôt obtenu, le crédit se donne de nouvelles limitations. L'activité est attribuable à une substance, il n'est pas dit qu'elle est la substance : première prudence. Elle est attribuable à une ou plusieurs substances : deuxième prudence. Et enfin, admirable accumulation de modalisations, "sans pour autant préjuger que la substance TRF ainsi mise en évidence correspond certainement au médiateur physiologique» (p. 2, 1. 44). Rien ne permet de dépenser le crédit indiscutable obtenu par cette Note en soutenant qu'est vrai dans le corps ce qu'on a prouvé dans le laboratoire : troisième prudence. La prudence est un stratagème, nous le savons depuis les Grecs, par lequel on se protège d'avance contre les attaques et par lequel, dans le cas présent, on donne discrètement une leçon de conduite scientifique à ceux qui, comme Shibuzawa, ont couru le risque de donner la composition chimique d'une substance à peine isolée. 
Dans cet article nous avons opéré deux transformations : une extension et une inversion. Nous avons étendu à un article de science exacte une analyse dont les concepts ont été forgés pour les textes littéraires; nous avons montré que la littérature scientifique est une partie de la littérature, invitant par là d'autres chercheurs à ne pas se laisser intimider par l'aspect rébarbatif des textes scientifiques. Toutefois il serait impossible d'élargir l'analyse que nous avons faite au " genre " scientifique (s'il existe) ; il ne s'est agi dans cette étude que d'un article et d'un seul. Le renversement consiste en ceci : on étudie toujours la science en termes spéculatifs comme une activité tournée vers la nature. Nous avons inversé ce rapport et considéré la production scientifique, en des termes plus militaires, comme une série d'opérations tournées vers le champ d'étude, lui-même identifié avec la littérature de frontière. Dans ce schéma la nature procure les munitions par lesquelles les coups sont rendus imbattables. Nous avons ainsi réintroduit des personnages que l'on croyait impossibles sur la scène théorique : le Stratège, le Droit, le Combat, le Vouloir et surtout la Rhétorique. Nous avons donc rempli l'une des conditions de la sociologie des sciences qui est de redécouvrir la société dans la rationalité, et, seule de toutes les sciences, le désordre sous l'ordre. Aussi simpliste et limitée que soit l'analyse qui précède, elle permet d'ouvrir trois directions pour la recherche qui ne fait que débuter. La première est directement littéraire : qu'est-ce qu'un bon ou un mauvais article scientifique ? Chaque chercheur actualise dans ses évaluations une « compétence ", au sens linguistique du terme, qui n’a jamais été mise au jour et qui définit à l'intérieur des sciences les plus quantitatives d'innombrables degrés de qualité. La seconde direction est plus proprement philosophique : qu'est-ce qu'une théorie et comment tracer une différence réelle entre la rhétorique et la démonstration ? Dans la mesure où l'on découvre de l'agonistique là où l'on attendait de la déduction, et de la production là où l'on attendait de la représentation, l'idée que les philosophes se font de la science et de leur propre discours théorique, doit se modifier quelque peu. La troisième enfin, directement sociologique, pose la question du crédit scientifique. L'économie de la science étudie comment le capital investi accroît la production ; la sociologie américaine des sciences, comment le capital symbolique (l'autorité) est accumulé par les savants ; l'épistémologie, comment l'argumentation devient forte ou faible. Il est temps d'étudier la science du point de vue d'une économie générale du crédit, en appelant crédit à la fois, le capitalargent, le capital-autorité, le capital-données et l'accréditation. C'est à l'étude de l'accumulation, de la circulation et de la transformation de ces différentes formes de crédit, que cet article prépare. 


\section{Post-scriptum(Actes de la recherche)}

Les objections qui ont été adressées à cette analyse par un des auteurs de l'article étudié et que Bruno Latour a bien voulu nous communiquer, se rangent sous trois chefs principaux :

fétichisme du texte :

l'article n'est qu'un moyen de transmission et n'a pas d'importance en tant que tel.

Ce qu'il y a finalement, sous les textes, c'est bien la Nature.

Les effets réels s'exercent sur d'autres corps, non sur des textes.

cécité à l'information transmise et au caractère concret de l'expérimentation :

On va conclure que nous avons rempli cinq pages pour dire ce qu'on aurait pu dire en deux lignes. Ce texte est au contraire bourré d'information.

Si l'on ne donne pas le détail des livres de protocoles, ce n'est pas pour cacher quoi que ce soit. C'est simplement inutile.

Les résultats donnés ne sont pas des “ jeux d'écriture "; il faut parler plutôt de “symboles" ou de " langues". Les résultats sont simplement traduits par des instruments ( ... ) nous n'avons pas d'autres moyens de les atteindre, mais les effets réels sont indépendants. Avec toutes les données nous construisons une image complète, cohérente.

vision naïvement cynique et agonistique des stratégies scientifiques et sous-estimation des obstacles épistémologiques:

tout est ramené à des motivations personnelles, comme si le scientifique était matois ou rusé. Nous étudions, nous ne jouons pas.

On découvre, on ne crée pas. De temps à autre, des rapports d'idées qui n'avaient pas été faits sont proposés. Nous construisons des " trucs". 
Combien de temps ne faut-il pas pour enlever un faux concept ? Combien d'années pour démolir petit à petit une erreur qui traîne?

\section{Notes}

* Nous en publions aussi la traduction vers l'anglais aux pages 115-135 de ce numéro [Note de la rédaction].

** Ce texte est reproduit aux pages 107 à 110 du présent numéro de Technostyle [Note de l'éditeur].

1 Cf. les études classiques de D. de Solla Price, Little Science-Big Science, New York, Columbia University Press, 1965 ; $\mathrm{cf}$. une récente application in C. Mullins, "The Development of a New Scientific Specialty ", Minerva, 10, 1972.

2 À la notable exception de J. Gusfield : "The Literary Rhetoric of Science ", American Sociological Review, 41 (1), fév. 1976. Mais cette étude porte sur une science "soft ", qui imite le style qu'elle croit être celui des sciences « hard ». De là les conclusions de l'auteur, tout à fait différentes des nôtres.

3 Nous faisons référence aux études classiques de G. Genette, Figure III, Paris, Éd. du Seuil, 1972 ; de É. Benveniste, Essais de linguistique générale, vol. 1, Paris, Éd. du Seuil, 1970 ; de A. Greimas, La sémantique structurale, Paris, Larousse, 1966.

4 Cf. A.J. Greimas, Sémiotique et sciences humaines, Paris, Ėd. du Seuil, 1976.

5 Un exemple remarquable dans M.J. Mulkay and D.O. Edge, "Cognitive, Technical and Social Factors in the Emergence of Radio Astronomy ", Information sur les sciences sociales 12(6), déc. 1973, pp. 25-71. Dans une optique complètement différente, cf. Michel Foucault, Les mots et les choses, Paris, Gallimard, 1966.

6 En particulier H. Zuckermann : « Patterns of Name Ordering among Authors of Scientific Papers ", American Journal of Sociology 74(3), nov. 1968.

7 Il faut signaler que les Comptes rendus ne publient que des Notes et non des articles. Ces notes n'ont pas d'autre but, selon le mot de l'auteur : " que d'intriguer le public scientifique pour qu'il soit presque sûr d'être convaincu et qu'il décroche son téléphone pour demander les renseignements qui manquent dans une Note aussi courte ".

8 L'article peut être lu tant qu'il se trouve à la frontière de la discipline. Quand il est intégré dans la science froide il n'a plus à être relu sauf pour vérifier des méthodologies, refaire l'histoire d'une discipline ou mesurer le chemin parcouru. Toutefois mème sans être lu ou relu il peut être cité. Le mot "utilisé " ou " dépecé » serait plus juste que " lu ». Il est possible grâce au Science Citation Index d'évaluer qui a utilisé l'article et pendant combien de temps. Les résultats sont les suivants : cet article a reçu quarante citations depuis 1964 dans des articles extérieurs au groupe et quatorze dans ceux du groupe. Onze articles confirment qu'il est bien le premier à avoir mis en évidence l'activité TRF, confirmation qui vient aussi bien des fondateurs du paradigme que des compétiteurs directs. Sept confirment le texte mais le citent avec ceux des auteurs dont il cherche à se distinguer. Parmi les cinq auteurs qui le citent pour des raisons techniques un seul cite un détail qui peut passer pour de l'information, le « débit de 50-60 ml/h », p. 1 (l. 29). 
9 Pour une récente présentation du problème en français, cf. Schally et Kastin, « Les hormones de l'hypothalamus », La recherche, 63, janv. 1976. Article intéressant parce qu'écrit par le compétiteur du groupe. L’histoire est différente, et même le nom donné aux substances.

$10 \mathrm{ll}$ ne faut pas confondre l'éponymie (par exemple : l'effet Compton) avec la persistance des modalités. L'une est le comble du prestige scientifique, l'autre une marque de critique ou de dérision. Ainsi quand on dit la " GHRH de Schally ne s'est pas montrée active dans nos mains " on veut dire qu'il n'y a là qu'une substance locale qui ne peut circuler comme GHRH. En ce cas le génitif est une marque de dérision.

11 Même plusieurs années après il est possible d'ouvrir les livres de protocoles et de recalculer les données brutes. Malgré l'idée dominante, l'énoncé scientifique n'est jamais libre de ses modalisations, contrairement à l'œuvre d'art qui les fait disparaître et à l'idéologie qui les maquille.

$12 \mathrm{Cf}$ un récent article de Chubin and Moitra, Content Analysis of References: Adjunct or Alternative to Citation Counting, Soc. St. of Sc, vol. 15, n. 4, nov. 1975, p. 423.

13 Cf. P. Bourdieu : « Le champ scientifique ", Acte de la recherche en sciences sociales, 2-3, 1976.

*** Ce texte est reproduit aux pages 112 et 113 du présent numéro de Technostyle [Note de l'éditeur].

14 Cette remarque, beaucoup trop grossière, n'est là que pour annoncer une étude à faire.

\section{Références}

Benveniste, E. (1970). Essais de linguistique générale (Vol. 1). Paris: Éditions du Seuil.

Bourdieu, P. (1976). Le champ scientifique. Actes de la recherche en sciences sociales, 2-3.

Chubin, D. \& Moitra, S. (1975). Content analysis of references: adjunct or alternative to citation counting. Social studies of science, 5 (4) 423-41.

Foucault, M. (1966). Les mots et les choses. Paris: Gallimard.

Genette, G. (1972). Figures III. Paris: Éditions du Seuil.

Greimas, A. (1976). Sémiotique et sciences humaines. Paris: Éditions du Seuil.

Gusfield, J. (1976). The literary rhetoric of science. American sociological review, 41 (1).

Latour, B., \& S. Woolgar. (1986). Laboratory life: the construction of scientific facts, second edition. Princeton, NJ: Princeton University Press. 
Mulkay, M. J. \& Edge, D. O. (1973). Cognitive, technical and social factors in the emergence of radio astronomy. Information sur les sciences sociales, 12 (6), 25 71 .

Mullins, C. (1972). The development of a new scientific specialty. Minerva, 10. Schally and Kastin (1976). Les hormones de l'hypothalamus. La recherche, 63. de Solla Price, D. (1965). Little science-big science. New York: Columbia University Press.

Zuckermann, H. (1968). Patterns of name ordering among authors of scientific papers. American journal of sociology, 74 (3). 
ENDOCRINOLOGIE. - Présence dans un extrait de tissus hypothalamiques d'une substance stimulant la sécrétion de lhormone hypophysaire thyréotrope (TSH). Première purification par filtration sur gel Sephadex. Note $\left(^{*}\right)$ de MM. Roger Guillemin Eilchi Yamazaki, Marian jutisz et EDVART SAKIZ, présentée par M. Robert Courrier.

I De nombreux résultats de Physiologie expérimentale sur le contrôle de la sécrétion de l'hormone thyréotrope ont conduit à postuler l'existence d'une neurohumeur d'origine hypothalamique qui serait le médiateur final, au niveau du parenchyme antéhypophysaire, de ce contrôle exercé par le diencéphale [voir la récente revue de Bogdanove (')]. Dans cette Note, nous montrons l'existence dans des extraits de l'hypothalamus d'une substance qui répond aux caractéristiques attendues de cette neurohumeur, que nous appellerons TRF (pour Thyrotropin Releasing Factor) par analogie avec CRF (Corticotropin Releasing Factor) et LRF (LH-

10 Releasing Factor). La méthode utilisée a conduit à une purification partielle de cette substance.

I 2 Plusieurs auteurs ont déjà dit avoir mis en évidence et purifié la substance TRF $\left[\left({ }^{2}\right),\left({ }^{3}\right),\left({ }^{4}\right),\left({ }^{6}\right)\right]$. Dans une revue récente $\left({ }^{5}\right)$ l'un de nous a exprimé ses réserves sur les conclusions de Shibusawa et coll. Les mêmes réserves

15 doivent s'appliquer à celles avancées par Libert $\left(^{6}\right)$. Reichlin $\left({ }^{7}\right)$ vient d'ailleurs de confirmer notre point de vue après avoir étudié le matériel en provenance du laboratoire de Shibusawa. Les résultats rapportés par Schreiber et coll. [( $\left.{ }^{3}\right)$, $\left.{ }^{(4)}\right]$ présentent plus d'intérêt; cependant, même dans ce dernier cas, n'ont pas été réunies toutes les conditions nécessaires ( $\mathrm{cf}$. infra) pour affirmer que la

20 fraction active de Schreiber et coll. n'agit que par stimulation de la sécrétion de TSH dans leurs expériences in vivo comme in vitro.

Matériel et méthodes. - Préparation des extraits. Filtration sur "Sephadex». - Pour chaque expérience Ca. $5 \mathrm{~g}$ d'un extrait acétique $2 \mathrm{~N}$ de 250 à 300 fragments d'hypothalamus de mouton préparé comme dans $\left({ }^{8}\right)$

25 sont repris dans le tampon acétate de pyridinium $0,1 \mathrm{M}, \mathrm{pH} 5$ et appliqués sur une colonne de «Sephadex G-25» $(5,5 \times 90 \mathrm{~cm})$, volume d'exclusion $640 \mathrm{ml}$. Toute la filtration se fait en chambre froide $\left(+4^{\circ} \mathrm{C}\right)$ à un débit de $50-60 \mathrm{ml} / \mathrm{h}$; on prélève 100 fractions de $20 \mathrm{ml}$ chacune. Une expérience identique est réalisée avec des quantités correspondantes de tissu du cortex 30 cérébral.

Bioétalonnages. - Pour l'étalonnage de la TSH, nous utilisons une méthode adaptée de la technique de McKenzie ( ${ }^{9}$ ) chez la Souris ou le Rat, normal ou hypophysectomisé, soumis à un régime pauvre en iode 
[p. 2]

et prétraité avec ${ }^{131}$ et thyroxine. Pour la mise en évidence de l'activité TRF, nous utilisons la même préparation mais avec des doses moindres de thyroxine. Les détails de cette technique avec une méthode nouvelle d'expression et d'analyse des résultats seront rapportés ailleurs. La vasopressine est mesurée par la méthode de Dekanski, l’ocytocine par la méthode de Coon, l'activité mélanophorétique par la méthode de Long et Guillemin ( ${ }^{5}$ ).

Resultats. - Deux zones d'activité (A et B, fig. 1) sont constamment retrouvées avec le test pour TSH sur l'ensemble des fractions obtenues à partir de l'hypothalamus. La première zone, $\mathrm{A}$, (fractions $\mathrm{T} 1$ à $\mathrm{T} 10, \mathrm{fig} .1$ )

10 correspond à des substances de poids moléculaire élevé non retenues sur "Sephadex G-25 "; cette activité persiste après hypophysectomie (Table 1); elle correspond donc probablement à l'hormone TSH ou à des substances pseudoTSH présentes dans l'hypothalamus. La deuxième zone d'activité, $B$, (fractions $T 41$ à $T$ 49, Table 2 ) correspond à des substances d'un poids 15 moléculaire relativement peu élevé ( $c f$. courbe des activités mélanophorétiques, fig. 1). Cette fraction B ( T 41 à T 49) n'est plus active après hypophysectomie (Table 3); nous avons montré par ailleurs qu'après hypophysectomie la réponse à la TSH est identique à celle obtenue chez l'animal normal, ('Table 4). L'activité de la fraction $B$ dépend de la concentration de thyroxine circulante (Table 5).

20 Elle est stable à la chaleur à la différence de la TSH (Table 6). L'hydrolyse $\begin{array}{lllll}\text { trypsique } & (\mathrm{pH} & 8, & 28{ }^{\circ} \mathrm{C}, & 24 \mathrm{~h})\end{array}$ ne détruit pas l'activité biologique de la fraction $\mathrm{B}$, mais cette activité disparaît après hydrolyse pepsique $\left(\mathrm{pH} 3,0,28^{\circ} \mathrm{C}, 24 \mathrm{~h}\right)$ ou acide $(\mathrm{HCl} 5,7 \mathrm{~N}$, $110^{\circ} \mathrm{C}, 24 \mathrm{~h}$ ). L'injection de doses multiples de la fraction $\mathrm{B}$ ( T 41 à $\mathrm{T} 49$ ) donne

25 des réponses qui sont une fonction linéaire du logarithme de la dose injectée (Table 2 ). La fraction $B$ ne produit pas de potentiation au niveau thyroïdien, d'une quantité connue de TSH injectée chez l'animal hypophysectomisé (Table 7). La fraction $B$ a été retrouvée constamment active dans plusieurs expériences de fractionnements d'extraits hypothalamiques

30 identiques à celle décrite ici. L'activité de cette fraction $B$ est localisée dans l'effluent en dehors des pics d'activité correspondant à vasopressine et ocytocine ( $f \mathrm{ig} .1$ ). Des doses massives de vasopressine et d'ocytocine n'ont pas donné chez le Rat de signes de stimulation hypophyso-thyroïdienne dans des conditions où, par ailleurs, la TSH est active

35 (Table 8). La fraction B n'a pas d'activités correspondant à ce qu'on a décrit sous le nom de "TSH retard " (long acting TSH). Aucun effet TRF n'a été trouvé dans l'effluent (fraction B) de la filtration sur "Sephadex" de l'extrait acétique du cortex cérébral.

g 6 Conclusions. - Les activité décrites plus haut et attribuables à une ou 40 plusiers substances localisées dans la zone B provenant d'un extrait hypothalamique, correspondent aux caractéristiques hypothétiques attendues de la neurohumeur TRF. Par facilité opérationnelle, nous parlerons donc de cette fraction B comme de la fraction TRF, sans pour autant préjuger 


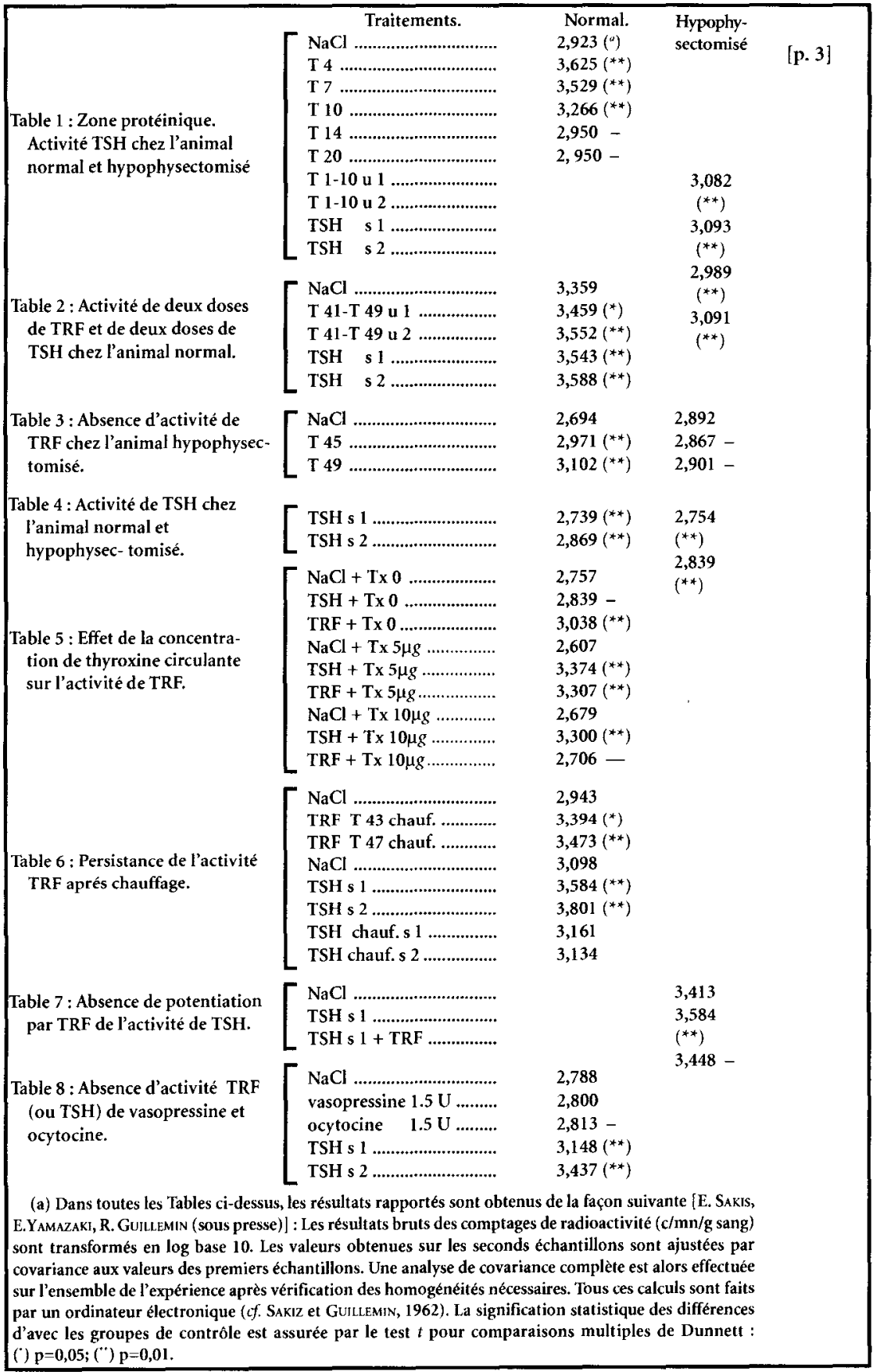




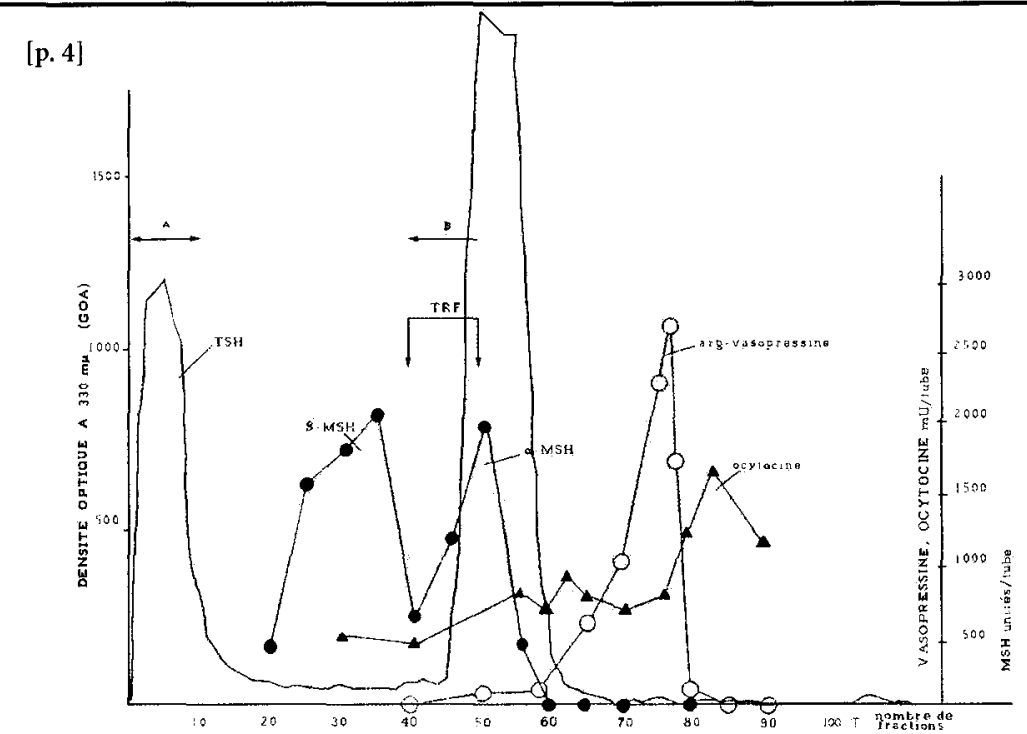

Fig. 1. Fractionnement d'un extrait acétique d'hypothalamus de mouton sur colonne de "Sephadex G-25 ". 4,9 g de l'extrait sont filtrés sur une colonne de $5,5 \times 90 \mathrm{~cm}$ équilibrée avec le tampon acétate de pyridinium $0,1 \mathrm{M}, \mathrm{pH} 5$. Débit de la colonne : 50 à $60 \mathrm{ml} / \mathrm{h}$. Volume des fractions : $20 \mathrm{ml}$. Des prises de $0,3 \mathrm{ml}$ de chaque fraction sont utilisées pour le dosage colorimétrique au biuret à $330 \mathrm{~mm}$ (J. GoA, Scand. J. Clin. Lab. Inv, 5 1953, P. 218; A. V. Schally et R. Guillemin, Texas Reports Biol. Med., 18, 1960, p. 133). Les fractions sont réunies deux par deux et lyophilisées. Pour le dosage de l'activité TRF, on utilise l'équivalent de $2,0 \mathrm{ml}$ de l'effluent original, par animal.

que la substance (TRF) ainsi mise en évidence correspond certainement au médiateur physiologique du contrôle hypothalamique de la sécrétion thyréotrope.

(*) Séance du 23 juillet 1962.

(1) E. M. Bogdanove, Fed. Proc, 21, 1962, p. 623.

(3) K. Shibusawa et al., Endocrinol. Japon., 6, 1959, p. 131.

(3) V. SCHREIBER et al., Experientia, 17, 1961, p. 264.

(4) V. Schreiber et al., Physiol. bohemoslov., 10, 1961, p. 417.

(5) R. Guillemin et A. V. Schally, Proc. U. S. P. H. International Neturoendocrinology Meeting, Miami, Flo. 1961, University of Chicago Press Publ.

(6) O. Libert, Histophysiologie du Complexe Hypothalamo-Hypophysaire, Paris, 1961 , p. 231-244 (dactyl.); Séminaires du Collège de France, Chaire d'Histophysiologie, $\mathbf{n}^{0} 1$.

(7) S. Reichlin, N. I. H. Conference on T. S. H., S. Werner, C. C. Thomas, Publ. Springfleld, Ill, 1962.

(8) R. Courrier et al., Comptes rendtus, 253, 1961, p. 922.

(9) J. M. MCKENZIE, Endocrinology, 63, 1958, p. 372.

(10) E. Sakiz et R. Guillemin, Comptes rendus, 254, 1962, p. 3743.

(11) Ces travaux ont été subventionnés par des fonds de recherches provenant du C.N.R.S. et du Ministère américain de la Santé (U.S. P. H.) ( ${ }^{\circ}$ A.5534).

(Laboratoire de Morphologie expérimentale et Endocrinologie, College de France.) 
comme le médiateur hypothalamique, correspondant à une certaine fonction antéhypophysaire ? C'est là une question d'importance primordiale pour évaluer la valeur ou la signification des nombreux rapports qui ont proposé telle ou telle substance comme une de ces neuro-hormones hypothalamiques. Et nous verrons que bien peu de ces rapports satisfont aux critères stricts que nous allons énoncer :

1. La préparation purifiée ou la substance pure en question doit provenir d'extraits de tissus de la région hypothalamique. (Ce qui n'élimine pas de facto une origine ubiquitaire ou multiple possible - particulièrement la neurohypophyse; cf. aussi les travaux de GUILLEMIN et coll. (1957) sur l'activité hypophysiotrope de la substance $P$ d'origine cérébrale ou intestinale.)

2. La substance en question (ou au moins l'activité hypophysiotrope correspondante) doit se retrouver dans le sang portal du système vasculaire hypothalamohypophysaire.

3. Elle doit, par définition, stimuler la sécrétion de l'hormone antéhypophysaire correspondante. On peut s'attendre, comme dans d'autres exemples de l'Endocrinologie classique, à ce que cette stimulation de la sécrétion antéhypophysaire se fasse (à l'intérieur de certaines limites) en suivant une fonction linéaire du log. de la dose injectée.

4. Puisque cette substance est l'information terminale et qu'elle doit agir directement sur l'antéhypophyse, on doit pouvoir démontrer son activité hypophysiotrope dans un système in vitro oủ l'hypophyse survit en dehors de toute connexion hypothalamique.

5. De même cette substance doit agir chez l'animal avec une lésion hypothal amique qui «bloque " la fonction hypophysaire (spontanée) correspondante, dans des conditions où la sensibilité périphérique à l'hormone antéhypophysaire n'est pas modifiée (cf. proposition suivante).

6. L'activité de cette substance doit disparaître chez l'animal hypophysectomisé, ce qui prouvera bien que l'activité est transhypophysaire. Encore faut-il montrer que dans les conditions expérimentales utilisées après hypophysectomie, la sensibilité périphérique ( $c f$. proposition $n^{\circ} 8$ ) à l'hormone hypophysaire correspondante est identique à celle de l'animal avec lésion hypothalamique où la substance est active. S'il n'en était pas ainsi, la disparition de l'activité après hypophysectomie pourrait être due non à la disparition d'un effet transhypophysaire mais à la baisse de sensibilité de l'effecteur périphérique après hypophysectomie, soit par effet direct (manque de la trophine hypophysaire correspondante) soit par effet indirect - manque d'une ou plusieurs hormones hypophysaires ou périphériques, impliquées d'une façon médiate et non immédiate (directe) dans le phénomène étudié - permissive action de INGLE (1954 a et $b$ ).

7. On s'attendrait à ce que la substance en question montre des variations dynamiques de sa concentration dans l'hypothalamus en fonction de différents états (endocriniens) induits expérimentalement. 
8. Comme il n'existe pas de méthodes de mesure directe des hormones antéhypophysaires dans les divers liquides biologiques, à la différence des stéroïdes ou des hormones thyroïdiennes, tous les résultats obtenus dans chacun des problèmes posés ci-dessus reviennent à des mesures indirectes de l'activité hypophysaire correspondante (par exemple: ACTH est "mesuré " par son effet sur la sécrétion de la corticosurrénale, TSH est "mesuré " par son effet sur la sécrétion de la thyroïde, etc.). Ce qui signifie que la substance en question doit être libre d'activité hormonale chez l'hypophysectomisé (cf. proposition $\mathrm{n}^{\circ} 6$ ). Tous ces tests de mise en évidence de l'activité hormonale hypophysaire doivent utiliser des critères périphériques indubitables chaque fois que cela est possible. Si les méthodes pour ce faire sont particulièrement laborieuses ou compliquées et si les expériences de routine utilisent des critères indirects, il faut montrer dans des expériences particulières que l'activité hypophysiotrope établie de routine sur les critères indirects (par exemple, mesure des concentrations en acide ascorbique de l'ovaire pour l'hormone $\mathrm{LH}$ ) se vérifie avec des critères directs et indubitables (par exemple déclenchement de l'ovulation et formation de corps jaunes pour l'hormone $\mathrm{LH}$ ). C'est la partie validation.

9. Le facteur hypophysiotrope doit augmenter la concentration plasmatique de l'hormone hypophysaire circulante. La difficulté technique actuelle de mesure des niveaux plasmatiques des diverses hormones antéhypophysaires rend d'ailleurs l'étude de cette proposition, particulièrement difficile.

10. De même doit-on montrer que la substance n'agit pas par potentiation périphérique de l'hormone antéhypophysaire. On peut en effet concevoir qu'une substance proposée comme un médiateur hypothalamique stimulant la sécrétion de telle hormone hypophysaire quand elle est injectée à l'animal d'expérience n'agisse que par potentiation d'une quantité fixe de cette hormone hypophysaire qui serait présente à tout moment : le résultat, dans nos tests périphériques, serait le même dans les deux cas. La question est résolue en montrant que le facteur hypothalamique étudié ne modifie pas l'effet d'une ou plusieurs doses de l'hormone hypophysaire correspondante quand il est injecté simultanément chezl'animal hypophysectomisé dans les conditions précisées plus haut (proposition $n^{\circ} 6$ ).

11. Dans le cas d'études in vitro il faut montrer que la substance en question agit par stimulation de la décharge de l'hormone hypophysaire considérée et non par potentiation périphérique (cf. proposition $\mathrm{n}^{\mathrm{0}} 10$ ) ou par inhibition d'une inactivation possible de l'hormone hypophysaire sécrétée par la glande dans le système in vitro.

12. Stimulant la sécrétion (décharge) d'une hormone antéhypophysaire, un facteur hypophysiotrope devrait faire diminuer le contenu hypophysaire en cette hormone, au moins dans certaines limites de temps après injection. En fait, l'hypophyse étant essentiellement une glande de stockage, il est difficile de mettre en évidence la disparition de quantités qui ne représentent que 0,1 à 1,0 p. 100 du stock hormonal total. Plus intéressantes seraient les informations qui auraient

R. Guillemin. « Sur la nature des substances hypothalamiques... ", Joumal de physiologie, 55, 1963. 
